\title{
ESPIRITUALIDADE E SAÚDE: Estudo Caso-Controle
}

\author{
Maria Beatriz Andrade Marques SILVA ${ }^{1}$ \\ Lorena Borges Cambraia Barreiros AMARAL ${ }^{1}$ \\ Rinaldo Borges de ALMEIDA ${ }^{1}$ \\ Soraya de Matos Camargo GROSSMANN ${ }^{2}$
}

\author{
${ }^{1}$ Médicos \\ ${ }^{2} \mathrm{MSD}, \mathrm{PhD},{ }^{2}$ docente da Universidade Vale do Rio Verde e Pontifícia Universidade Católica
}

Recebido em: 12/09/2016 - Aprovado em: 11/12/2016 - Disponibilizado em: 18/12/2016

\begin{abstract}
RESUMO
A relação entre espiritualidade, religião e saúde tem sido cientificamente estudada, avaliada e documentada. Os objetivos do trabalho foram identificar a influência da espiritualidade no processo de saúde, verificar se a espiritualidade influencia, positivamente, a saúde do indivíduo que nela acredita e comparar se o profissional de saúde tem a mesma espiritualidade da população em geral. $\mathrm{O}$ trabalho foi do tipo caso-controle, a partir de uma amostra por conveniência, em municípios da região metropolitana de Belo Horizonte. A amostra de 261 indivíduos foi dividida em dois grupos: (Grupo 1 - casos) profissionais da saúde, 92 indivíduos e (Grupo 2 - controle), população geral, 169 indivíduos, correspondentes a 35,3\% e 64,7 \% da amostra, respectivamente. Os sujeitos da pesquisa responderam um questionário sobre a influência da espiritualidade e o impacto da mesma no status de saúde. Foram realizadas medidas de associação e risco através do cálculo do OR (Odds Ratio) e do valor de $p$ e foram considerados resultados válidos quando o valor de $p$ foi $\leq 0,05$. Todas as mulheres responderam que a espiritualidade é importante, assim como, $100 \%$ dos indivíduos $\geq 60$ anos de idade; 255 indivíduos, 97,7\% dos participantes, acreditam que a espiritualidade é importante na vida do indivíduo. Houve significância estatística quando se analisou a questão sobre o conhecimento de alguma cura milagrosa e se o indivíduo tem com quem falar sobre assuntos espirituais, apontando que os profissionais de saúde acreditam menos nas curas milagrosas e a população geral tem mais dificuldade em encontrar com quem falar sobre assuntos espirituais, $\mathrm{p}<0,001 \mathrm{e} \mathrm{p}=0,035$, respectivamente. Pode-se considerar que os dois grupos pesquisados e analisados têm similaridades quanto à espiritualidade e a relacionam de maneira positiva com o status de saúde. As diferenças justificam a necessidade de mais pesquisas sobre a relação entre espiritualidade e saúde, e entre os profissionais da saúde e a população em geral.
\end{abstract}

Palavras-chave: Espiritualidade; saúde; profissionais.

\begin{abstract}
The relationship between spirituality, religion and health have been scientifically studied, evaluated and documented. The research aimed to identify the influence of spirituality on health process, verify if spirituality influences positively the health of the individual who believes in it and compare if the health professional has the same spirituality of the general population. The study was case-control, from a convenience sample in the metropolitan region of Belo Horizonte, Brazil. The sample of 261 individuals was divided into two groups: (Group 1 - cases) health professionals, 92 individuals, and (Group 2 - control), the general population, 169 individuals, representing 35.3\% and $64.7 \%$ of the sample, respectively. The subjects answered a questionnaire about the influence of spirituality and its impact on their health status. The measures of the association and risk were made by calculating the OR (Odds Ratio) and of the value of $p$. The results were considered valid when the $p$ value was $\leq 0.05$. All of the women answered that spirituality is important as well as $100 \%$ of the individuals $\geq 60$ years of age; 255 subjects, $97.7 \%$ of the participants believe that spirituality is important in their lives. There was statistical significance when analyzing the question about the knowledge of some miraculous cure and if the individual has someone whom to talk about spiritual matters, pointing that health professionals believe less in miraculous healings and the general population has more difficulty in finding someone to talk about spiritual matters, $p<0.001$ and $p=0.035$, respectively. One can consider that the two groups analyzed have similarities regarding spirituality and believe in a positive relationship between it and their health status. The differences justify the need for more research on the relationship between spirituality and health and between health professionals and the general population.
\end{abstract}

Keywords: Spirituality; health; professionals. 


\section{Introdução}

A religião é a forma mais antiga de prática médica. Os Xamãs eram os "homens sábios", curadores da tribo, cujos tratamentos eram rituais religiosos (SULMASY, 2009). Atualmente, a espiritualidade é estudada de forma mais independente e livre relacionando-se a práticas, tais como: meditação, relaxamento, e quaisquer outras técnicas que têm por objetivo estabelecer um contato mais íntimo entre o praticante, da mesma, e sua alma.

A relação entre espiritualidade e saúde tem sido cientificamente estudada, avaliada e documentada (KOENIG, 2004). O termo espiritualidade esteve, de maneira geral, associado à religião. Pode-se definir espiritualidade "como um sistema de crenças que enfoca elementos intangíveis, que transmite significado e sentido a eventos da vida". Enquanto a espiritualidade tem caráter transcendente, individual e autodeterminada, a religião tipicamente envolve conexões com crenças e rituais compartilhados de uma comunidade (SAAD, 2001). Mesmo aquelas pessoas que não se classificam como religiosas se consideram espiritualistas de alguma maneira (SULMASY, 2009).

Nesse contexto, a espiritualidade tem sido considerada como uma importante faceta da qualidade de vida (QV)-saúde observada e reconhecida pela Organização Mundial de Saúde (OMS), em vários de seus documentos como forma de assistência à saúde, a fim de se alcançar o bem-estar humano nos níveis físico, mental e espiritual (PAHO/WHO, 2000).

A busca de sentido, significado ou propósito para a realidade de nossas vidas nos remete a um cuidado maior com os vários aspectos do viver humano, inclusive, com a saúde em todos os níveis e dimensões. O momento e o tema são propícios para as pesquisas e vasto é o campo.

A relevância do tema se deve ao interesse crescente pelo assunto a partir da segunda metade do século passado, em especial a partir da década de 80 . No meio científico não foi diferente, somente nos últimos vinte e seis anos foram publicados 3.409 artigos, dos quais 390 foram artigos de revisão. Espiritualidade e saúde são dois aspectos da vida humana que estão ganhando importância na sociedade do século XXI. A saúde pode ser beneficiada através da prática da espiritualidade.

Os objetivos do trabalho foram identificar a influência da espiritualidade no processo de saúde, verificar se a espiritualidade influencia, positivamente, a saúde do indivíduo que nela acredita e comparar se o profissional de saúde tem a mesma espiritualidade da população em geral.

\section{Metodologia}

O presente trabalho foi do tipo casocontrole, a partir de uma amostra por conveniência, em municípios da região 
metropolitana de Belo Horizonte. Os indivíduos foram divididos em dois grupos: profissionais da área da saúde (Grupo 1 casos) e população geral (Grupo 2 - controle). Para a validação dos resultados o número de controle representou quase duas vezes o total de casos incluídos. Os sujeitos da pesquisa responderam a um questionário (Apêndice I) sobre a influência da espiritualidade e o impacto da mesma no status de saúde.

As respostas às perguntas foram diretas tendo como opções as respostas positivas ou negativas, sendo que e a última pergunta permitia distinguir se o indivíduo pertencia ou não ao grupo dos profissionais da saúde. $\mathrm{O}$ questionário utilizado foi baseado nas perguntas sugeridas por um Painel de Consenso do American College of Physicians - American Society of Internal Medicine (Lo et al., 1999). Todos os sujeitos que participaram da pesquisa assinaram o Termo de Consentimento Livre e Esclarecido (Apêndice II) e possuíam idade igual ou superior a 18 anos, à época da aplicação do questionário.

As perguntas do questionário foram codificadas para a análise dos dados como mostra o Quadro 2. Os dados obtidos foram compilados em um banco de dados utilizando o programa Excel, e a análise estatística foi realizada usando o programa EPI-INFO 7.0. Foram realizadas medidas de associação e risco através do cálculo do OR (Odds Ratio) e do valor de $p$. Foram considerados resultados válidos quando o valor de $p$ foi $\leq 0,05$.

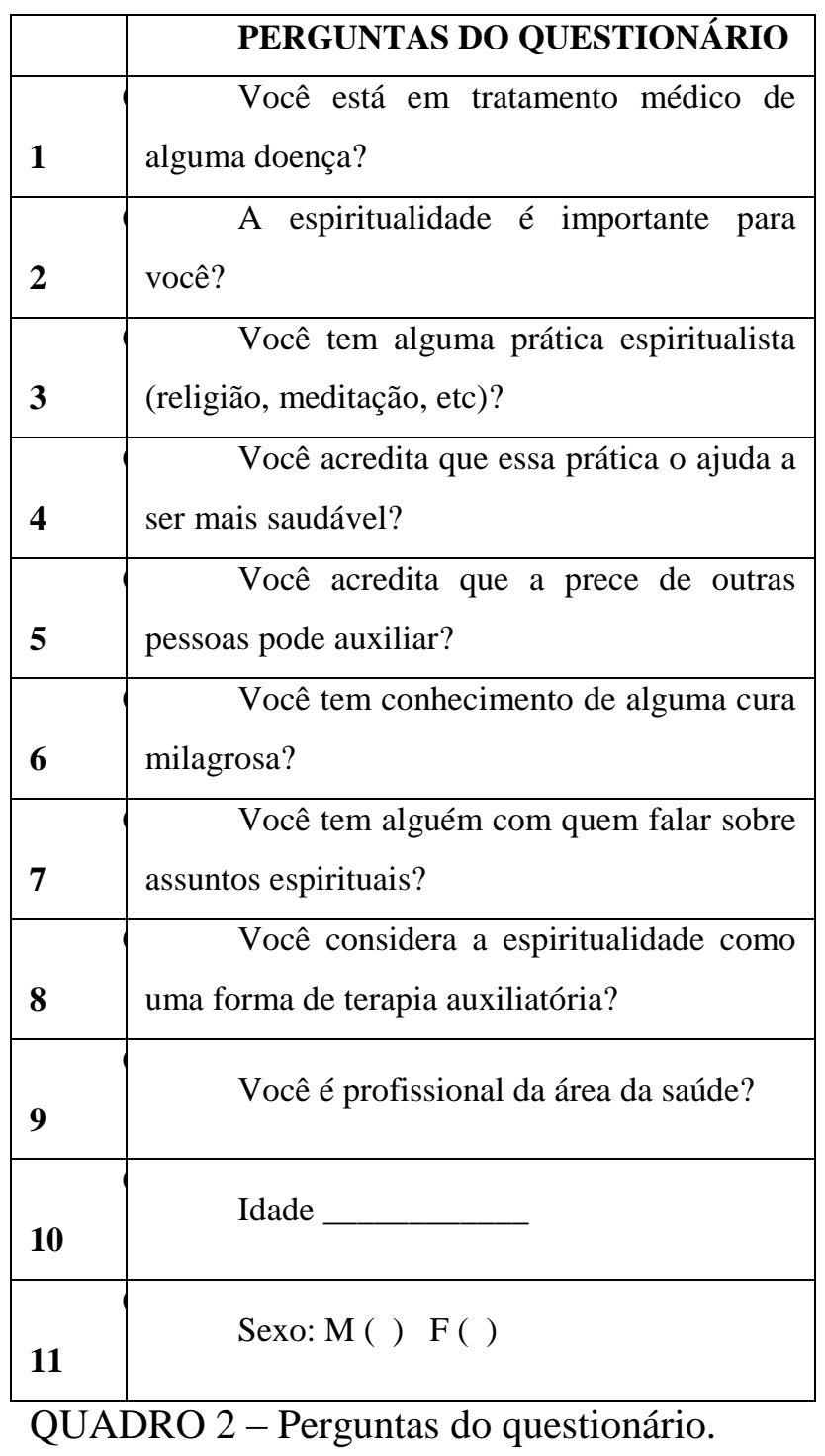

\section{Resultados}

Um total de 261 indivíduos, responderam ao questionário: $92(35,3 \%)$ do GRUPO 1 e $169(64,7 \%)$ do GRUPO 2. Para a análise dos dados foram considerados três grupos de faixa etária: $108 \quad(41,4 \%)$ indivíduos entre 18 e 30 anos de idade; 119 $(45,6 \%)$ entre 31 e 59 anos e $34(16,0 \%)$ acima de 60 anos; sendo $109 \quad(41,8 \%)$ 
participantes foram do sexo masculino e 152 $(58,2 \%)$ do sexo feminino.

A maior parte dos indivíduos respondeu positivamente a todas as perguntas (Gráfico $1)$.

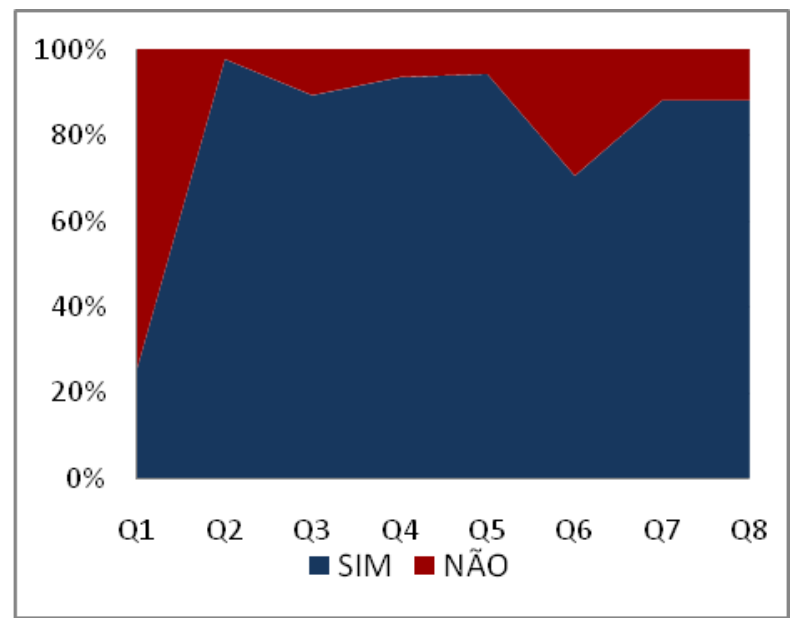

Gráfico 1: Respostas positivas e negativas às perguntas

Todas as mulheres responderam que a espiritualidade é importante (Q2) enquanto 94,2\% dos homens responderam positivamente. Quando analisado a idade, 104 (96,3\%) indivíduos com idade entre 18 e 30 anos, $117(98,3 \%)$ entre 31 e 59 anos e 34 $(100 \%) \geq 60$ anos, responderam da mesma maneira, apontando que $255(97,7 \%)$ dos indivíduos participantes da pesquisa acreditam que a espiritualidade é importante para a vida do indivíduo.

Quando as respostas foram analisadas individualmente os achados mostraram que as perguntas: a espiritualidade é importante para você? (Q2), você acredita que esta prática o ajuda a ser mais saudável? (Q4) e você acredita que a prece de outras pessoas pode auxiliar? (Q5), foram respondidas positivamente. Em relação à pergunta, você está em tratamento de alguma doença? (Q1), $194(74,33 \%)$ dos participantes responderam negativamente, isto é, não se encontravam em tratamento médico no momento da pesquisa (Gráfico 2).

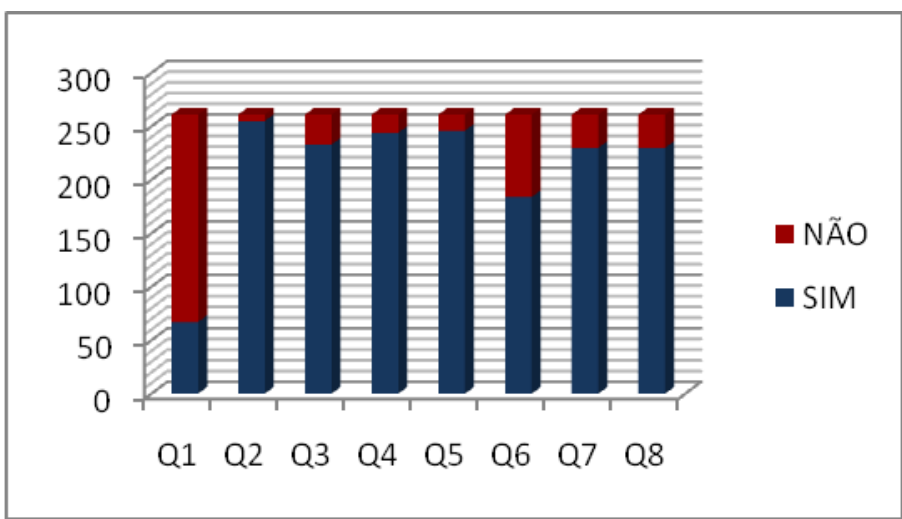

Gráfico 2: Análise individual de cada pergunta

A análise comparativa entre as perguntas de Q2, Q3, Q4 e Q8 em relação à pergunta Q9, se o profissional era da área da saúde ou não, mostrou resultados semelhantes nos dois grupos (Tabela 1). 
TABELA 1 - Comparação entre perguntas e respostas do Grupo 1 (profissionais da saúde) e Grupo 2 (outros profissionais).

\begin{tabular}{|c|c|c|c|c|c|c|}
\hline Amostra & & Grupo 1 & & rupo 2 & & \\
\hline RESPOSTAS & $\begin{array}{c}\text { POSITIVA } \\
\text { n }(\%)\end{array}$ & $\begin{array}{l}\text { NEGATIVA } \\
\text { n (\%) }\end{array}$ & $\begin{array}{c}\text { POSITIVA } \\
\text { n }(\%)\end{array}$ & $\begin{array}{l}\text { NEGATIVA } \\
\text { n (\%) }\end{array}$ & $p$ & OR \\
\hline Q2 & $91(98,9)$ & $1(1,1)$ & $164(97,1)$ & $5(2,9)$ & 0,31 & \\
\hline Q3 & $83(90,2)$ & $9(9,8)$ & $150(88,8)$ & $19(11,2)$ & 0,45 & \\
\hline Q4 & $85(92,4)$ & $7(7,6)$ & $159(94,1)$ & $10(5,9)$ & 0,39 & \\
\hline Q5 & $85(92,4)$ & $7(7,6)$ & $161(95,3)$ & $8(4,7)$ & 0,25 & \\
\hline Q6 & $50(54,6)$ & $42(45,7)$ & $134(79,3)$ & $35(20,7)$ & $<0,001$ & 0,31 \\
\hline Q7 & $86(93,5)$ & $6(6,5)$ & $144(85,2)$ & $25(14,8)$ & 0,035 & 2,48 \\
\hline Q8 & $83(90,2)$ & $9(9,8)$ & $147(87,0)$ & $22(13,0)$ & 0,28 & \\
\hline
\end{tabular}

\section{Discussão}

Houve significância estatística quando se analisou a questão Q6 (você tem conhecimento de alguma cura milagrosa?) para o Grupo 1, apontando que os profissionais de saúde acreditam menos nas curas milagrosas.

Em relação a Q7 (você tem com quem falar sobre assuntos espirituais?) observou-se que o Grupo 2, tem mais dificuldade em encontrar com quem falar sobre assuntos espirituais que o Grupo 1.
Há evidências da existência de uma relação positiva entre espiritualidade/religiosidade e saúde já comprovada cientificamente. Essa relação se manifesta através da saúde mental, física e social, por exemplo, menos depressão, menos abusos de substâncias, menos doenças cardiovasculares e maior longevidade (KOENIG, 2004; CURLIN, 2007; HASSED, 2008, ROCHA, 2011). A resposta positiva para as perguntas Q3 e Q4, pela maioria dos 
participantes (cerca de 90\%), confirma esses achados da literatura.

Muitos pacientes gostariam que o seu médico ou profissional de saúde levasse em consideração a sua espiritualidade, inclusive, para a escolha do tratamento (SAAD, 2001, HASSED, 2008). As perguntas sugeridas pelo Painel de Consenso do American College of Physicians são questões simples e abertas para estimular a conversa com o paciente, não sugerindo que o profissional da saúde deva fazer o papel de um pastor ou padre (LO et al, 1999). De acordo com os resultados encontrados no trabalho, 255 (98\%) dos participantes responderam positivamente à pergunta se a espiritualidade era importante para eles (Q2). Quando se comparou a pergunta Q2 com ambos os sexos e com os três grupos de faixa etária, a espiritualidade foi mais importante no sexo feminino e na faixa etária com 60 anos ou mais, os quais tiveram $100 \%$ de respostas afirmativas.

Em relação à pergunta (Q7), 230 (88\%) dos participantes disseram que têm alguém com quem falar sobre os assuntos espirituais, apontando para o fato de que as pessoas estão receptivas para conversar sobre o tema. Apesar disso, 93\% dos profissionais no Grupo 1 responderam positivamente a essa pergunta confrontando com cerca de $85 \%$ da população participante do Grupo 2 ( $p$ 0,035 - OR 2,48), sugerindo uma lacuna que pode e deve ser preenchida pelo profissional da saúde, haja vista a sua maior facilidade em ter alguém com quem falar sobre assuntos espirituais. A prática dessa oportunidade de conversa pode ser um daqueles momentos especiais e únicos, quando se constrói uma relação profissional de confiança (KOENIG, 2004). Pode-se suspeitar que a dificuldade maior dos integrantes do Grupo 2 em ter com quem conversar sobres assuntos espirituais, do que os profissionais da saúde, se deve ao fato do tema estar, geralmente, relacionado a misticismo e tabus, gerando desconforto e permanecendo um campo vasto de opiniões divergentes.

Quando perguntados se acreditavam que a prece de outras pessoas poderia auxiliar (Q5) e se eles acreditavam que a espiritualidade poderia ser uma forma de "terapia auxiliatória" (Q8), os profissionais do Grupo 1 e a população do Grupo 2 responderam positivamente a Q5 (92 e 90\%, respectivamente) e a Q8 $(95$ e $87 \%$, respectivamente). Alguns trabalhos demonstraram uma associação positiva entre a prece e melhores resultados de saúde (JANTOS e KIAT, 2007; GUIMARÃES, 2007), salientando sua importância e possível influência na melhoria da saúde.

Com relação ao conhecimento de alguma "cura milagrosa" (Q6), houve diferença entre os resultados. No Grupo 1, $54 \%$ dos profissionais conhece alguma cura milagrosa, já no Grupo 2 a prevalência foi de cerca de 79\% ( $p<0,001-$ OR 0,31). Esse resultado pode ser decorrência do fato de que 
o Grupo 1, profissionais da área da saúde, analisam com olhar cético o assunto "cura milagrosa", muitas vezes considerando cura somente aquelas comprovadas cientificamente. Pode-se inferir que essas diferenças ocorrem em relação ao Grupo 1, por ser esse constituído de profissionais que, supostamente, buscam fundamentar seus conceitos e opiniões em bases cientificamente comprovadas e uma "cura milagrosa" pode gerar dúvidas nesse quesito.

As semelhanças entre os dois grupos pesquisados são a provável evidência, e podem ser um marcador, da semelhança entre os seres humanos quando comparados em suas necessidades mais essenciais, isto é, a busca de um contato mais próximo com o ser transcendente que habita em cada um.

As diferenças encontradas são a marca da individualidade e do livre arbítrio que nos é dado a cada renascimento, o sagrado direito da escolha. As semelhanças nos tornam um com o Todo e as diferenças nos permitem ser o Um, exercitando-se.

As diferenças justificam a necessidade de mais pesquisas sobre a relação entre espiritualidade e saúde, e entre os profissionais da saúde e a população em geral, no sentido de buscar o entendimento de "por que”, como um ser humano, a espiritualidade é importante para ele. Sobretudo, tentar elucidar o fato de que o indivíduo acredita que "a prece de outras pessoas pode ajudar", no entanto, como profissional da saúde ele tem dificuldade em reconhecer a espiritualidade se manifestando como uma "cura milagrosa".

Pode-se considerar que os dois grupos pesquisados e analisados têm similaridades quanto à espiritualidade e a relacionam de maneira positiva com o seu status de saúde.

Com base nas informações obtidas nesse trabalho, e corroboradas pela literatura científica, fica evidenciada a necessidade, que se apresenta como uma oportunidade, do profissional de saúde utilizar a espiritualidade do seu paciente como mais uma ferramenta a favor da prevenção da saúde em todos os níveis, fortalecendo a definição de saúde dada pela OMS "saúde é um estado de completo bem-estar físico, psicossocial e espiritual".

Tratar do tema espiritualidade com o paciente toma poucos minutos do tempo da consulta médica, e a informação adquirida e o ganho na relação médico-paciente, em termos de construção de confiança, pode fazer diferença na melhoria na saúde do indivíduo.

Considerando que a espiritualidade é importante para a maioria da população, e que poucos estudos, analisaram objetiva e estatisticamente, novos estudos fazem-se necessários. Dessa forma, poderemos confrontar, os novos achados, buscando sempre respostas para perguntas ainda não bem respondidas. Talvez dessa maneira, consigamos comprovar cientificamente um assunto, tido como subjetivo, e praticar a Medicina baseada em evidências. 


\section{REFERÊNCIAS}

1. AMARAL, L. "O Divertimento e a Dor": como é possível curar o corpo, recuperando o espírito, na Nova Era? Motriz. 5(2), 1999.

2. ELIAS, A.C.A. et al. Programa de treinamento sobre a intervenção terapêutica "relaxamento, imagens mentais e espiritualidade" (RIME) para re-significar a dor espiritual de pacientes terminais Rev. Psiq. Clín. 34, supl 1; 6072, 2007.

3. CURLIN, A.F. et al. Religion, Spirituality, and Medicine: Psychiatrists' and Other Physicians' Differing Observations, Interpretations, and Clinical Approaches. Am J Psychiatry. 164(12):1825-1831, 2007.

4. GUIMARÃES, H.P. e AVEZUM, Á. O Impacto da Espiritualidade na Saúde Física. Rev. Psiq. Clín. 34, supl 1; 88-94, 2007.

5. HASSED, C. The role of spirituality in medicine. Australian Family Physician. 37(11), nov., 2008.

6. JANTOS, M.; KIAT, H. Prayer as medicine: how much have we learned? MJA. 186: S51-S53, 2007.

7. KOENIG, H.G. Religion, Spirituality and Medicine: Application to Clinical Practice. JAMA. 284; 13, oct., 2000.

8. Religion, Spirituality and Medicine: Research Findings and Implications for Clinical Practice. Southern Medical Journal. 97; 12, dec; 2004.

9. LO, B; QUILL; T; TULSKY, J. Discussing palliative care with patients. Ann Intern Med. 130(9); 744-749, 1999.
10. LUCHETTI, G. et al. Espiritualidade na prática clínica: o que o clínico deve saber? Rev Bras Clin Med. 8 (2): 154-8, 2010.

11. $\mathrm{PAHO} / \mathrm{WHO}$. BUILDING BLOCKS: Comprehensive Care Guidelines for Persons Living with HIV/AIDS in the Americas. SUMMARY REPORT. Jun. $2000.16 \mathrm{p}$.

12. PAIVA, J. Religião, enfrentamento e cura: perspectivas psicológicas. Estudos de Psicologia. 24(1); 99-104, jan-mar, 2007.

13. PANZINI, R.G. et al. Qualidade de Vida e Espiritualidade. Rev. Psiq. Clín. 34, supl 1; 105-115, 2007.

14. BANDEIRA,D.R. Coping (enfrentamento) Religioso/Espiritual. Rev. Psiq. Clín. 34, supl 1; 126-135, 2007.

15. PAULA, E.S.; NASCIMENTO, L.C.; ROCHA, S.M.M. Religião e espiritualidade: experiência de famílias de crianças com Insuficiência Renal Crônica. Rev Bras Enferm. 62(1): 100- 6, jan-fev, 2009.

16. PERES, M.F.P. et al. A importância da integração da espiritualidade e da religiosidade no manejo da dor e dos cuidados paliativos. Rev. Psiq. Clín. 34, supl 1;82-87, 2007

17. PESSINI, L. e BERTACHINI, L. Novas Perspectivas em Cuidados Paliativos: ética, geriatria, gerontologia, comunicação. O Mundo da Saúde. 29(4), 2005.

18. Terminalidade e Espiritualidade: uma reflexão a partir dos Códigos de Ética Médica brasileiros e a leitura comparada de alguns países. O Mundo da Saúde. 33(1); 35-42, 2009.

19. RIZZARDI, C.D.L. et al. Espiritualidade e religiosidade no enfrentamento da dor. O Mundo da Saúde. 34(4):483-487, 2010. 
20. ROBERTS, J.A. et al. Factors influencing views of patients with gynecologic cancer about end-of-life decisions. Am J Obstetr Gynecol.176; 166-72, 1997.

21. ROCHA, N.S. e FLECK, M.P.A.

Avaliação de qualidade de vida e importância dada a espiritualidade/religiosidade/crenças pessoais (SRPB) em adultos com e sem problemas crônicos de saúde. Rev. Psiq. Clín. 38 (1); 19-23, 2011.

22. RUMBOLD, B.D. A review of spiritual assessment in health care practice. MJA. 186(10): S60-S62. 2007.

23. SAAD, M. et. al. Espiritualidade baseada em evidências. Acta Fisiátrica. 8(3); 107 112, 2001.

24. SANCHEZ, Z. vd M. e NAPPO, S.A. A Religiosidade, a Espiritualidade e o Consumo de Drogas. Rev. Psiq. Clín. 34. 34(1); 73-42, 2007.

25. SEARIGHT, H.R. e GAFFORD, J.

Cultural Diversity at the End of Life: Issues and Guidelines for Family Physicians. American Family Physician. 71; 3, feb., 2005.

26. SOUZA, J.C. e SOARES, A. Resenha do livro: Espiritualidade e Qualidade de Vida. Rev. Psiq. RS. 27(2); 218-219, 2005.

27. SULMASY, D.P. Spirituality, Religion, and Clinical Care. CHEST. 135;6, jun, 2009. 